\title{
LSPR-Based Label Free Sensing in Absorbance Mode: Sensing of Therapeutic Antibodies in Serum ${ }^{\dagger}$
}

\author{
Kristian Göeken ${ }^{1}$, Tom Brinkman 1, Loes Segerink ${ }^{2}$, Thijs Oude-Munnink ${ }^{3}$, Ron Gill 1,4,* \\ and Aleksandar Andreski 5 \\ 1 Nanobiophysics Group, University of Twente, 7500 AE, Enschede, The Netherlands; \\ k.l.goeken@utwente.nl (K.G.); t.brinkman@student.utwente.nl (T.B.) \\ 2 BIOS Group, University of Twente, 7500 AE, Enschede, The Netherlands; 1.i.segerink@utwente.nl \\ 3 Department of Clinical Pharmacology, MST Hospital, 7512 KZ. Enschede, The Netherlands; \\ T.oudeMunnink@mst.nl \\ 4 Nanobio Lectorate, Saxion University of Applied Sciences, 7513 AB, Enschede, The Netherlands \\ 5 Nanophysics Lectorate, Saxion University of Applied Sciences, 7513 AB, Enschede, The Netherlands; \\ a.andreski@saxion.nl \\ * Correpondence: r.gill@saxion.nl \\ + Presented at the 5th International Symposium on Sensor Science (I3S 2017), Barcelona, Spain, \\ 27-29 September 2017.
}

Published: 4 December 2017

Gold nanoparticles support localized surface plasmons, which are coherent oscillations of the metal conductance electrons. While surface plasmon polaritons of gold films (used in surface plasmon resonance (SPR)-based sensing) require special methods to match the energy and momentum (such as the use of prisms or grating), localized surface plasmon resonance (LSPR) of gold nanoparticles can be directly excited by light propagating in free space.

The position of the surface plasmon resonance peak can be interrogated by measuring either transmitted light (absorbance mode) or scattered light (scattering mode). In both modes the position of the plasmon peak is known to be sensitive to the refractive index of the medium in the close vicinity of the nanoparticle's surface. Although the scattering peaks shifts more than the absorbance peak when the refractive index of the medium changes, absorbance measurements are more compatible with instrumentations available at any biochemistry lab, such as UV-VIS absorbance spectrometers.

The detection of antibodies in a complex media (cell culture media or human serum) is an important analytical problem with both biotechnological applications (in the case of recombinant antibodies producing cells, for example) and medical applications (in the case of allergy detection or immunotherapy supervision).

Here we present our results on the label-free detection of antibodies in complex media. We show that sub-nM concentration of antibodies can be detected, both in buffer and in complex media. We report on the effect of dilution of the complex media with buffer and we compare the use of gold nanoparticles in solution vs. surface bound gold nanoparticles. We then apply this method for the detection of Infliximab (a therapeutic antibody used for the treatment of autoimmune disease) in full serum.

(C) 2017 by the authors. Licensee MDPI, Basel, Switzerland. This article is an open access article distributed under the terms and conditions of the Creative Commons Attribution (CC BY) license (http://creativecommons.org/licenses/by/4.0/). 